\title{
The relationship between latitudinal light variation and orbit and cranial size in humans
}

\begin{abstract}
Increased orbit size is suggested to be an adaptation for enhanced visual acuity and sensitivity in conditions of reduced light quality. Whilst light ambience has a well established correlation with eye size in birds and primates evidence in humans is very limited. The aim of this study was to analyze the anatomical compensations of the eye and visual cortex as a result of varying levels of light exposure. It was hypothesized that humans of higher latitudes will have an increased orbit size to improve visual sensitivity and acuity in conditions of decreased light, and thus greater cranium size due to enlarged visual cortices. Craniometric measurements of 1,209 male and 1,021 female individuals from 27 series coming from different latitudes were sourced from William W. Howells Craniometric Data Set. Mean cranial and orbit size was calculated by combining linear craniometric measurements of length, width and height for individual males and females at each latitude. Linear regressions of orbit and cranial size on latitude were created and significance was measured using Pearson's $r$ and $\mathrm{P}$ value. Partial correlations were calculated to test whether orbit size correlates with latitude independent of cranial size. Significant positive correlations were found between
\end{abstract}

i. Orbit and cranial size and

ii. Orbit and latitude size and

iii. Cranial size and latitude in males and females.

Additionally partial correlation values for latitude and orbit size were significant in both males and females. The relationship between visual system size and increasing latitude among humans is currently understudied. Significant relationships between visual system size and increasing latitude suggest that enlarged eyes were an evolutionary mechanism for individuals with compromised light availability. Other factors related to varying geographic location may also play a role.

Keywords: eye size, illuminance, visual acuity, visual sensitivity, males, females
Volume I Issue I - 2017

\author{
Alice Short \\ Department of Anthropological and Comparative Anatomy Unit, \\ University of Adelaide, Australia
}

\author{
Correspondence: Alice Short, department of Anthropological \\ and Comparative Anatomy Unit, University of Adelaide, \\ Australia, Tel +6I-452-567-987, \\ Email alice.short@student.adelaide.edu.au
}

Received: September 26, 2016 | Published: March 24, 2017

\section{Introduction}

Light ambience has a well established correlation with eye size in birds and primates. Studies performed on primates have discovered that nocturnal species have significantly larger orbit size when compared with diurnal species. ${ }^{1,2}$ The increased orbit size can be seen as an accommodation for enlarged eyes, an adaptation to allow enhanced visual acuity and sensitivity in conditions of lesser light quantity and quality. ${ }^{2}$ Studies comparing the avian eye with activity levels and habits discovered that nocturnal birds have significantly greater eye volume when compared to those of diurnal nature. ${ }^{1}$ This is possibly a compensation mechanism in response to low light levels, as a larger eye allows for an increased number of photoreceptive cells and hence more light to be received by the retina. ${ }^{1}$ Light gathering ability or visual sensitivity is determined by the size of corneal diameter. Enlargement of the cornea allows an increased pupil diameter which increases the amount of light that can enter the eye. Another important quality of eyes is visual acuity the ability to differentiate two shapes from one another. An increased axial length creates a larger retinal image which improves the ability to resolve to different objects and thus enhances visual acuity. ${ }^{3}$ Heightened light sensitivity also occurs as a result of increased in retinal summation. ${ }^{4}$ Retinal summation describes the number of photoreceptor cells for the number of bipolar cells in the retina and is an adaptation that occurs to increase light sensitivity. In the case of high-summation, a greater ratio of photoreceptor cells synapse to bipolar cells which allows for response to weak stimuli. ${ }^{5}$ Increased retinal summation results have been observed to in primates of nocturnal nature when compared to diurnal species. ${ }^{5}$ However, increased retinal summation in order to heighten light sensitivity comes at a price of reduced visual acuity $^{5}$ Visual acuity is negatively impacted under high-summation as individual light rays are indistinguishable to the brain. ${ }^{5}$ It has been suggested that an overall increased eye size would result in an improvement in both an increase in visual sensitivity and acuity. ${ }^{3}$ An eye with a greater axial length and corneal diameter is capable of perceiving more light and detail when compared to individuals with smaller eyes. ${ }^{3}$ However due to large variations in environment there is no ideal eye design rather that humans are expected to optimize eye shape for their specific needs of their environment. ${ }^{3}$ This knowledge can be applied to humans living in varying regions of the world. The amount of total light on the Earth's surface (luminance) decreases as day length decreases. ${ }^{4}$ Regions of higher latitude experience greater polarities of both minimum and maximum day length. Whilst average day length of countries remains the same throughout the world, the unequal distribution of daylight at regions of greater latitude results in periods of extended darkness during wintertime ${ }^{4}$ and thus activity of retinal photoreceptors varies accordingly. ${ }^{6}$ There are three different ranges of vision that are each activated by different levels of 
illuminance. Scotopic vision relates to vision at low light levels, such as nighttime and overcast days, and involves only rod activation. ${ }^{4}$ Photopic vision is active at high levels of light (e.g. daylight) by which vision are mediated by cones. ${ }^{4}$ Mesopic vision involves the activation of both rods and cones during periods of early twilight or moonlight. ${ }^{4}$ Locations of low latitude experience more day lengths with greater illuminance ${ }^{4}$ and thus habitants are largely active during photopic conditions, increasing the level of light exposure to the eye and maximizing cone activity ${ }^{4}$ contrastingly those in locations of increased latitude will have greater levels of activity during mesopic and scotopic conditions. ${ }^{4}$ The human orbit does not contain the eyeball alone. However along with the lacrimal apparatus and surrounding muscles, it is considered to accurately reflect the size of the adult eye.? A study on human orbits has confirmed a positive relationship between orbital and eyeball volume independent of brain size, thus suggesting the measurement of the human orbit a reliable measure of eye size. ${ }^{7}$ Eye size and the underlying functional meaning behind its variation have received little attention in humans. The aim of this study is to analyze the anatomical compensations that occur to the eye and brain as a result of varying levels of light exposure. It is hypothesized that humans from higher latitudes will have an increased orbit size to improve visual sensitivity and acuity in conditions of decreased light and thus greater cranium size due to enlarged visual cortices.

\section{Methods}

Craniometric measurements from 1,209 male and 1,021 female skulls originating from a range of 27 latitudinal points were sourced from William W. Howells Craniometric Data Set. ${ }^{8}$ Calculations of anthropometric points were performed to find the cranial and orbital size for each individual as per below formulas. The mean cranial and orbit size was then calculated for both males and females of each country. All latitudinal points were recorded using Google Latitude.

$$
\begin{aligned}
& \text { Cranial size }=\frac{G O L+X C B+B B H}{3} \\
& \text { Orbit size }=\frac{O B H+O B B}{2}
\end{aligned}
$$

Linear regressions were performed for 1 ) orbit size vs cranial module; 2) cranial module vs latitude and 3) orbit size vs latitude.

To test the whether orbit size was changing independently of cranial size, the partial correlation was calculated whilst holding cranial size constant as per below formula.

$$
r_{x y . z}=\frac{r_{x y}-r_{x z} r_{y z}}{\sqrt{\left(1-r_{x z}^{2}\right)\left(1-r_{y z}^{2}\right)}}
$$

\section{Results}

Significant correlations were found between orbit size of individuals and latitude in both male and female cohorts $(\mathrm{r}=0.285$, $\mathrm{p}<0.01 ; \mathrm{r}=0.312, \mathrm{p}<0.01$ respectively). Additionally significant positive correlations occurred between individual cranial and orbit sizes in both males $(\mathrm{r}=0.408,<0.01)$ and females $(\mathrm{r}=0.426, \mathrm{p}<0.01)$. Finally a significant positive relationship occurred between individual cranial size and latitude for males $(r=0.276, p<0.01)$ and females $(\mathrm{r}=0.282, \mathrm{p}<0.01)$. The partial correlation between orbit size and latitude whilst holding cranial size constant displayed a significant coefficient of correlation for females $(\mathrm{r}=0.221, \mathrm{p}<0.01)$ and males $(\mathrm{r}=0.193, \mathrm{p}<0.01)$.

\section{Discussion}

Both male and female data displayed significant partial correlations between latitude and orbital size, independently of cranial size $(\mathrm{r}=0.193, \mathrm{p}<0.01$ and $\mathrm{r}=0.221, \mathrm{p}<0.01$ respectively). This suggests that orbit size increases independently from brain size at regions of greater latitude. These data support previous findings by ${ }^{4}$ where significant interactions between orbital size latitude were also deemed independent of cranial size. The correlation between both male and female orbit size and increasing latitude was significant $(\mathrm{r}=0.285, \mathrm{p}<0.01, \mathrm{r}=0.312, \mathrm{p}<0.01$ respectively) Figure 1 . These data provide a strong argument to suggest that and enlarged orbit result from a developmental mechanism to enhance visual image quality of individuals thus supporting the hypothesis. An increase in orbit size can be attributed to an enlarged cornea thus increasing pupil diameter. ${ }^{7}$ This consequentially increases the amount of light gathering and thus improving visual sensitivity. ${ }^{7}$ Increased axial length and hence an increase in retinal image size will improve visual acuity. ${ }^{3}$ Enlarged orbit size will increase retinal summation, therefore increasing the number of photoreceptor cells in the eye. These adaptations result in an increased eye size and improve visual detection of weak stimuli in poor light conditions. ${ }^{3}$ These data can be supported in both avian and primate models, which attribute increased orbit size to decreased light quality. ${ }^{1-3}$ The significant partial correlation figures for males $(\mathrm{r}=0.193, \mathrm{p}<0.01)$ and females $(\mathrm{r}=0.221, \mathrm{p}<0.01)$ provide a strong indication that orbit size increases independently of cranial size, rather than enlarged eyes being simply a morphological consequence of an enlarged brain.

Latitude vs Average Orbit Size

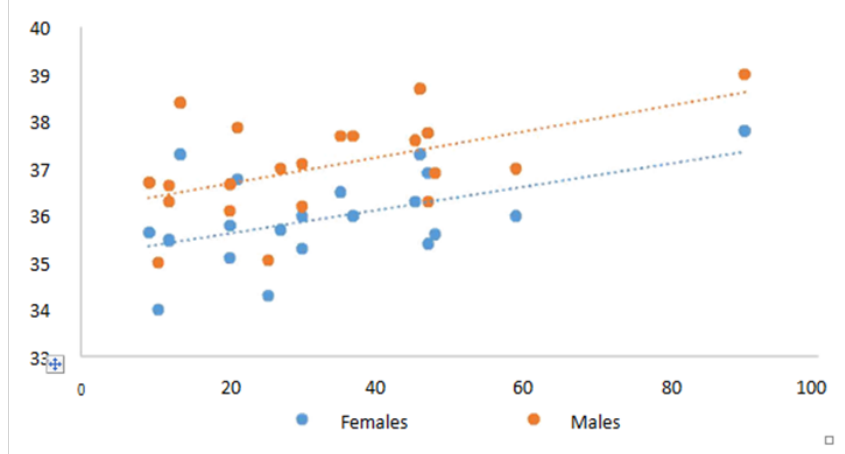

Figure I Correlation of orbit size with increasing latitude in females and males.

Significant positive relationships between cranial and orbit size were observed in both male $(\mathrm{r}=0.59, \mathrm{p}=0.0044)$ and female cohorts $(\mathrm{r}=0.72, \mathrm{p}=0.00034)$. These results may suggest the development of larger visual cortices to allow for greater neural pathways and connections as a result of the increasing number of photoreceptors in the retina. This can be seen as a co-evolutionary mechanism in order to achieve behavioral adaptations of increased visual sensitivity and acuity. This would therefore prove useful in environments of decreased illuminescence. Given that all anthropometric data used here date back to pre and early history, prior to the invention of electrical light ${ }^{9}$ individuals would not have survived in regions of high latitude had they not developed the physiological adaptions to assist in eye sight. Poor eye sight in these conditions of low light levels would have led to decreased ability to work, commute; self protect, farm and prepare food. Among hunters and gatherers individuals with 
heightened visual sensitivity and acuity would have improved chance of survival by greater ability to protect one self from predators. It can therefore be suggested that this increase in orbital and cranial size was a mechanism to maximize the amount of information gathered from the retinal image and thus improve visual perception by humans in regions where there is minimal to no light for extended periods of the year.

A significant positive relationship between cranial size and latitude was found in both males $(\mathrm{r}=0.276, \mathrm{p}<0.01)$ and females $(\mathrm{r}=0.282$, $\mathrm{p}<0.01)$ Figure 2. These data are supported by the findings of ${ }^{10}$ and Pearce (2007) both of whom have documented similar correlations of increased cranial size at greater latitudinal points. The independent increase of orbit size with latitude suggests that increased eye size may at part explain the reason for increased cranial volume. One study tested whether the positive scaling of orbital volume is associated with visual cortex volume in humans, in which a significance correlation was found. ${ }^{7}$ This supports the second part of the hypothesis, which predicted that increased cranium size would be observed at higher latitudes due to an increased visual system size.

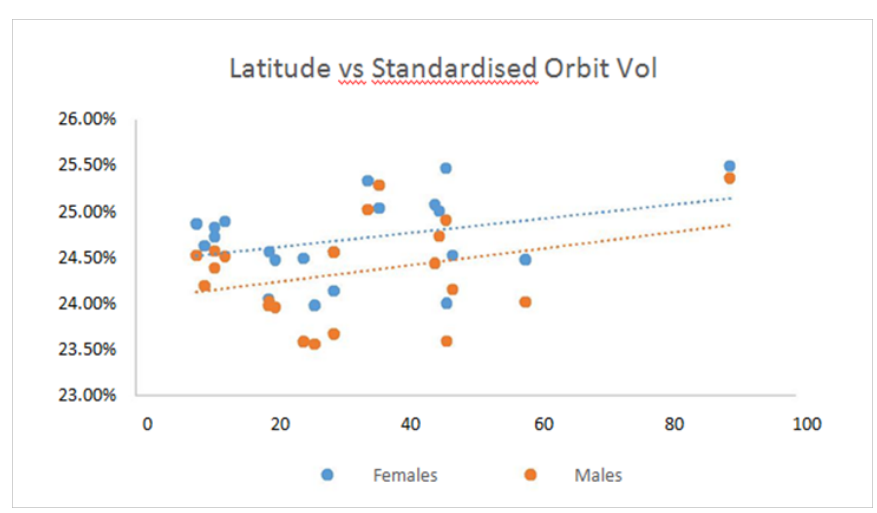

Figure 2 Increasing orbit volume with latitude when standardized against cranial volume.

Whilst the relationship between visual system size and latitude has been deemed significant in a study by Pearce \& Dunbar, the results in are limited by a small sample size of 55 skulls across 12 populations. The large sample size in this analysis (2,230 individual skulls across 22 latitudinal points) provides strong reliability which can support these previous findings. One limitation of this investigation is the inability to distinguish whether an increase in cranial size is due to enlarged visual cortices, or rather that of already established variables such as the decrease in temperature or vitamin D exposure. Additionally variation in diet amongst communities at different latitudinal points may have an effect on total brain size. Therefore, rather than measuring the entire cranium an alternate method for analyzing visual cortex size could be electro physical monitoring of the visual cortex using electroencephalogeny (EEG). This may eliminate this limitation in future studies by allowing measurement of activity specific to the visual cortex rather than that of the entire brain. Suggestions for future research into the relationship between orbit and cranial size and varying latitudes would be to undergo measurements of modern humans after the establishment of artificial light.

\section{Conclusion}

Whilst the correlation between orbit and cranial size at varying levels of light has been well documented in other species the relationship among humans is understudied. An increase in eye size assists in the improvement of visual acuity and visual sensitivity qualities that are increasingly important in environments where light quality and availability is reduced. Significant relationships between both orbit and cranial size with increasing latitude were discovered supporting the hypothesis that visual system size increases with increasing latitude. This is proposed to be an evolutionary mechanism in order to increase chances of survival in regions of compromised light availability.

\section{Acknowledgements}

I would like to thank Professor Maciej Henneberg for consultation and assistance with statistical methods and formatting of this manuscript.

\section{Conflict of interest}

Author declares there is no conflict of interest in publishing the article.

\section{References}

1. Garamszegi LZ, Moller AP, Erritzoe J. Coevolving avian eye size and brain size in relation to prey capture and nocturnality. Proc Biol Sci. 2002;269(1494):961-967.

2. Kay RF, Kirk EC. Osteological evidence for the evolution of activity pattern and visual acuity in primates. Am J Phys Anthropol. 2000;113(2):235-262.

3. Hall MI, Ross CF. Eye shape and activity pattern in birds. Journal of Zoology. 2007;271(4):437-444.

4. Pearce E, Dunbar R. Latitudinal variation in light levels drives human visual system size. Biol Lett. 2012;8(1):90-93.

5. Veilleux CC, Kirk EC. Visual acuity in mammals: effects of eye size and ecology. Brain Behav Evol. 2014;83(1):43-53.

6. Schubert EF. Human eye sensitivity and phometric quantities. Cambridge University Press, Cambridge, UK; 2006. p. 275-289.

7. Pearce E, Bridge H. Is orbital volume associated with eyeball and visual cortex volume in humans? Ann Hum Biol. 2013;40(6):531-540.

8. Howells WW. Cranial variation in man. A study by multivariate analysis of patterns of difference. Among recent human populations. Peabody Museum of Archaeology and Ethnology.1973;67:1-259.

9. Sonnebom L. The Electric Light. Thomas Edison's Illuminating Invention, Infobase Publishing, New York, USA; 2007.

10. Ash J, Gallup GG. Paleoclimatic Variation and Brain Expansion during Human Evolution. Hum Nat. 2007;18(2):109-124. 